\title{
Wireless Transmission Detection and Monitoring System using GNU Radio and Multiple RTL - SDR Receivers
}

\author{
Madhuram Mishra*, Dr. Anjali Potnis** \\ *M.Tech. Student (Department of Electrical and Electronics Engineering, National Institute of Technical \\ Teachers Training and Research, Bhopal - 02 \\ ** Professor (Department of Electrical and Electronics Engineering, National Institute of Technical Teachers \\ Training and Research, Bhopal - 02
}

\begin{abstract}
Low cost signal detection and monitoring system is designed in this paper. Wireless Transmission Monitoring is required by security agencies in order to check transmissions taking place in a particular area. In this paper Wireless Transmission Monitoring System is implemented using GNU Radio and RTL - SDR device. The implemented system is capable to monitor signals of GSM Transmission, Commercial FM Broadcast; the system is developed using Software Defined Radio based RTL - SDR hardware. Monitoring System can also be extended as a signal receiver by making certain additions in the designed model. In this paper Multiple RTL SDR device based signal detection system is also designed. FM signals were successfully detected by the system in laboratory environment.

Keywords: Software Defined Radio, Digital Signal Processing, GNU Radio, RTL - SDR, Signal Detection, Transmission Monitoring, Multiple RTL - SDR
\end{abstract}

\section{INTRODUCTION}

Software Defined Radios (SDRs) are very exciting advancement in radio technology. The SDR uses software processing to perform most of the tasks that only hardware can perform in hardware based radio receivers. The hardware section of SDR system provides raw data to computer for further processing [8]. This powerful fusion of hardware and software has allowed SDR to be compact and have great functionalities than traditional hardware radio setup [9]. Transmission Detection and Monitoring System is designed using GNU Radio. The designed system is based on RTL - SDR device which is capable to detect signal in the frequency range 25 to $1700 \mathrm{MHz}$, GNU Radio based design are easy to implement [2]. The system is designed on GNU Radio Live Disk Ubuntu OS based Environment.

The GNU Radio based system is designed for wireless signal detection and monitoring in frequency range $50 \mathrm{MHz}$ to $1050 \mathrm{MHz}$, the RTL SDR supported frequency range is $25 \mathrm{MHz}$ to $1750 \mathrm{MHz}$, designed system can be extended to monitor frequency range of $25 \mathrm{MHz}$ to $1750 \mathrm{MHz}$ by making suitable changes in the software section.

Two RTL - SDR device are connected with the computer system and both the system are programmed to function simultaneously. The system can also be used to lock a particular frequency for continuous detection and other Source section to perform scanning at other frequency.
RTL - SDR device is used as Software Defined Radio hardware. Hardware based radio systems have very less capabilities limited just to receive particular signals only and physical interventions required to make changes in the system [3]. Using Software Defined Radio a reconfigurable system is implemented which can be used for wireless signal detection applications. Many devices are developed recently using SDR, such devices include RTL SDR Realtek based Software Defined Radio [4]. This device has 2 main ICs Raphael Micro R820T radio tuner and Realtek RTL2832U which is having USB data pump and ADC with 8-bit resolution. These SDR dongle are primarily developed to receive Terrestrial Television Signals. Due to efforts of SDR community, support package developed for RTL - SDR dongles to use them as Software Radio Hardware. RTL - SDR device can be used only to receive radio signals, for testing purpose we have used low cost FM transmitter and also used Raspberry Pi based FM transmitter [1]. RTL - SDR device is connected with the computer using USB Extension cable, in order to reduce noise due to computer system in the device [5]. Circuit diagram and high level block diagram of RTL - SDR is given in figure 1 . 


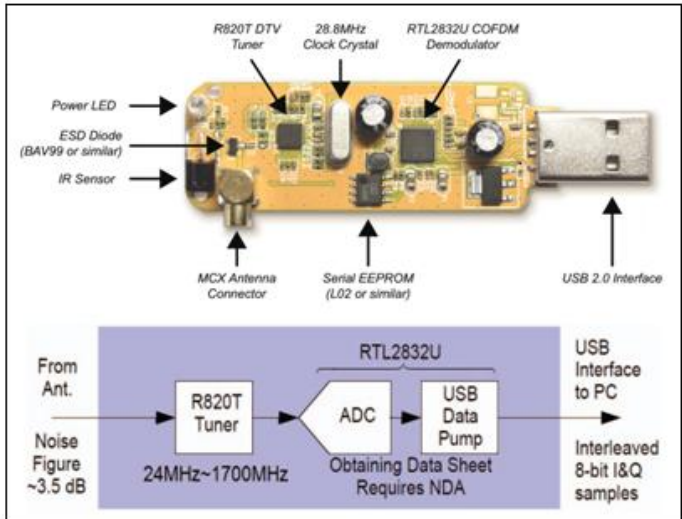

Figure 1 RTL - SDR Circuit and Block Diagram

\section{SIGNAL DETECTION AND MONITORING SYSTEM}

\section{A. Detection System using SDR}

In this paper wireless signal detection and monitoring system is designed using Software Defined Radio supporting hardware. The main advantage of proposed system is that ability of monitoring two frequencies at the same time by interfacing two RTL - SDR device. The system is developed by making suitable arrangements in GNU Radio to interface more than one USB device for SDR applications. Block diagram shown in Figure 2.

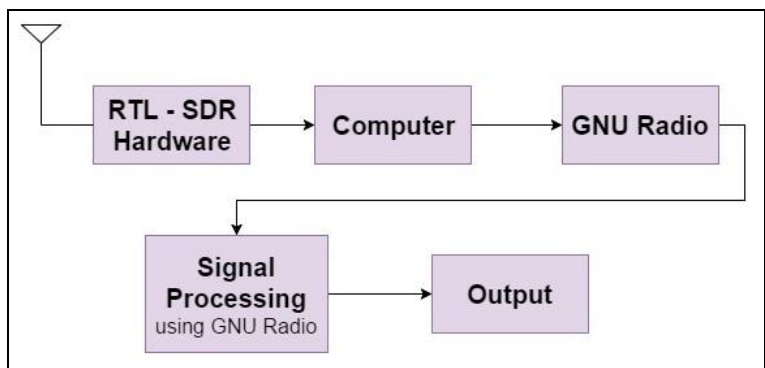

Figure 2 SDR device connection block diagram

\section{B. GNU Radio based detection and monitoring system}

GNU Radio provides options to design Software Defined Radio projects through simple block adding features. Beginners can easily start working on SDR and signal processing systems even without prior computer programming knowledge using GNU Radio. There are changes made in the Device Arguments Field of the RTL - SDR block in GNU Radio in order to use two USB devices for signal processing operations. Device Arguments: rtl=0 for source " 1 " and Device Arguments: $r t l=1$ for source " 2 ". Multiple SDR system diagram shown in Fig. 3.

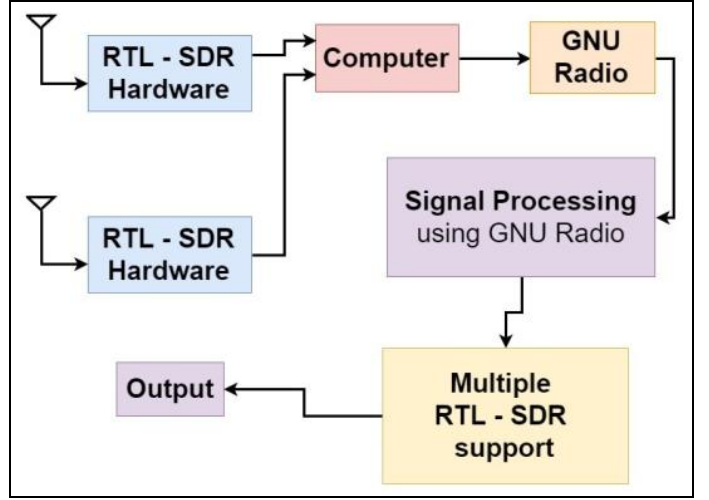

Figure 3 Multiple SDR Connection

RTL - SDR Source block is used to interface hardware with the computer system, two RTL SDR Source blocks are used. Both the blocks perform signal receiving function simultaneously. Changes made in Device Argument field in order to make the complete system suitable to work with more than one RTL - SDR dongle. After Source block FFT Sink block is connected. The sink block is used to view graphical results for the Frequency provided at the source block.

Detection system is also designed with option to monitor one frequency signal at a time as shown in the figure $2 \& 9$. Single RTL - SDR Source block is used and Waterfall Sink is connected with the source block. There is also option added to change the frequency through slider between $50 \mathrm{MHz}$ to $1050 \mathrm{MHz}$. Using slider frequency value of the RTL - SDR Source block can be changed while using the detection system. Using Waterfall Sink block various options available for enhancing graphical results obtained. Similar wireless signal detection and monitoring system is designed using two RTL - SDR Sources with options to get Time Scope and Waterfall plots for the received signals. The GNU Radio Companion basic flowchart diagram is shown in the figure 4 for detection system. 


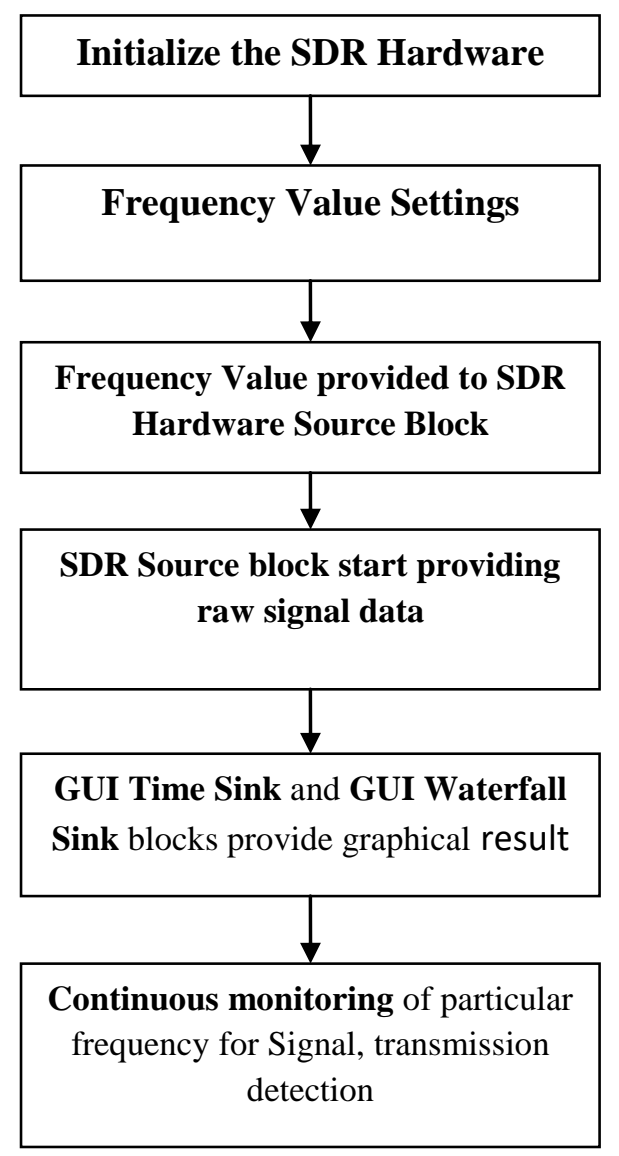

Figure 4 Flowchart for basic detection system

First the initialization of the RTL - SDR hardware is completed by providing the sample rate values. Frequency value can also be directly provided during initialization. Second frequency value can be provided by using any GUI element if required. The frequency value is accessed by the source block, the source block starts providing raw data to the computer system through RTL - SDR hardware connected at USB port. The data is processed using the waterfall sink block to provide result about the wireless signal at the particular frequency. The above steps are basic procedure required to follow in order to work with SDR source blocks and hardware using GNU Radio.

For two RTL - SDR system, Frequency values are provided using Slider or Text Box for RTL - SDR Source "1" and RTL - SDR Source " 2 ". The frequency values stored in variable accessed by the RTL - SDR Source block and particular frequency is set for respective source block, frequency values can also be directly provided in the Frequency Field of the RTL - SDR Source block. In order to pair RTL - SDR source block with a particular SDR, Device Arguments field is made different for two different source blocks as shown in the flow chart fig 8 " $r t l=0$ " and " $r t l=1$ " for source 1 and source 2 respectively.GUI Waterfall Sink and GUI Time Sink blocks are connected after source block to get graphical results about any signal present in the particular previously set frequency. Continuous signal monitoring can be performed at a particular frequency. There is option to change frequency for one source block without disturbing signal detection (monitoring) frequency of the other source block. GNU Radio blocks used in RTL SDR based Signal Detection and Monitoring System and their basic working discussed below.

\section{RTL - SDR Source}

For controlling RTL - SDR device through GNU Radio the source block is used. The frequency value for the detection is provided through this block. While using two RTL - SDR Source block the Device Argument value is changed as shown in figure 5. In the figure 5 Source block at left is used when more than one hardware is controlled using the model.

RTL-SDR Source
Device Arguments: rtl=0
Sample Rate (sps): $32 \mathrm{k}$
Ch0: Frequency $(\mathrm{Hz}): 89.9 \mathrm{M}$
Ch0: Freq. Corr. (ppm): 0
Ch0: DC Offset Mode: Off
Ch0: IQ Balance Mode: Off
Ch0: Gain Mode: Manual
Ch0: RF Gain (dB): 10
Ch0: IF Gain (dB): 20
Ch0: BB Gain (dB): 20

RTL-SDR Source Sample Rate (sps): $32 \mathrm{~K}$ Cho: Frequency $(\mathrm{Hz}): 98.3 \mathrm{M}$ Cho: Freq. Corr. (ppm): 0 Ch0: DC Offset Mode: Off Ch0: IQ Balance Mode: Off Ch0: IQ Balance Mode: Ch0: RF Gain (dB): 10 Cho: IF Gain (dB): 20 Cho: BB Gain (dB): 20
Figure 5 SDR Source Block in GNU Radio

\section{Sink Block (for obtaining Signal Graphs)}

Various Sink blocks in GNU Radio are available. In the design FFT Sink, Time Sink and Waterfall Sink blocks are used. While selecting the block only suitable type block can be used, if WX type model is designed then only WX type blocks can be used. Sink blocks diagram given in figure 6 .

\begin{tabular}{l}
\multicolumn{1}{c|}{ WX GUI FFT Sink } \\
Title: FFT Plot \\
Sample Rate: $32 \mathrm{k}$ \\
Baseband Freq: 0 \\
Y per Div: $10 \mathrm{~dB}$ \\
Y Divs: 10 \\
Ref Level (dB): 0 \\
Ref Scale (p2p): 2 \\
FFT Size: $1.024 \mathrm{k}$ \\
Refresh Rate: 15 \\
Freq Set Varname: None
\end{tabular}

QT GUI Time Sink Number of Points: $1.024 \mathrm{k}$ Sample Rate: $32 k$ Autoscale: No

QT GUI Waterfall Sink FFT Size: $1.024 \mathrm{k}$

Center Frequency (Hz): 0 Bandwidth $(\mathrm{Hz}): 32 \mathrm{k}$
Figure 6 - Sink Blocks used in model

\section{Slider Block (for changing frequency values)}

GUI Slider block is used in model for single RTL - SDR based system to change 
frequency of operation while the detection system is working. Figure 7 gives details about Slider Block. Maximum and minimum values are provided while using the block.

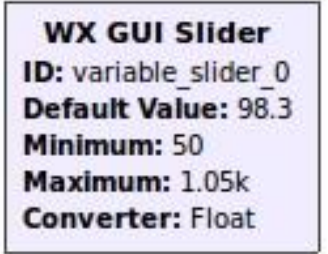

\begin{tabular}{|c|c|}
\hline \multicolumn{2}{|c|}{ Slider Block } \\
\hline $\begin{array}{l}\text { Default } \\
\text { Value }\end{array}$ & 98.3 \\
\hline Minimum & 50 \\
\hline Maximum & 1050 \\
\hline $\begin{array}{l}\text { Converter } \\
\text { Type }\end{array}$ & Float \\
\hline
\end{tabular}

Figure 7 Slider block used and its details

Working of multiple RTL - SDR hardware system is explained using Flowchart in figure 8. While implementing signal detection system only two RTL - SDR devices used. Both the RTL - SDR device were controlled by GNU Radio. Frequency value is provided directly in the GNU Radio block Frequency Field and not through any GUI element like Slider or Textbox. Detection system with single RTL - SDR Support is shown in figure 9 and Detection System designed using GNU Radio for two RTL - SDR shown in figure 10 .

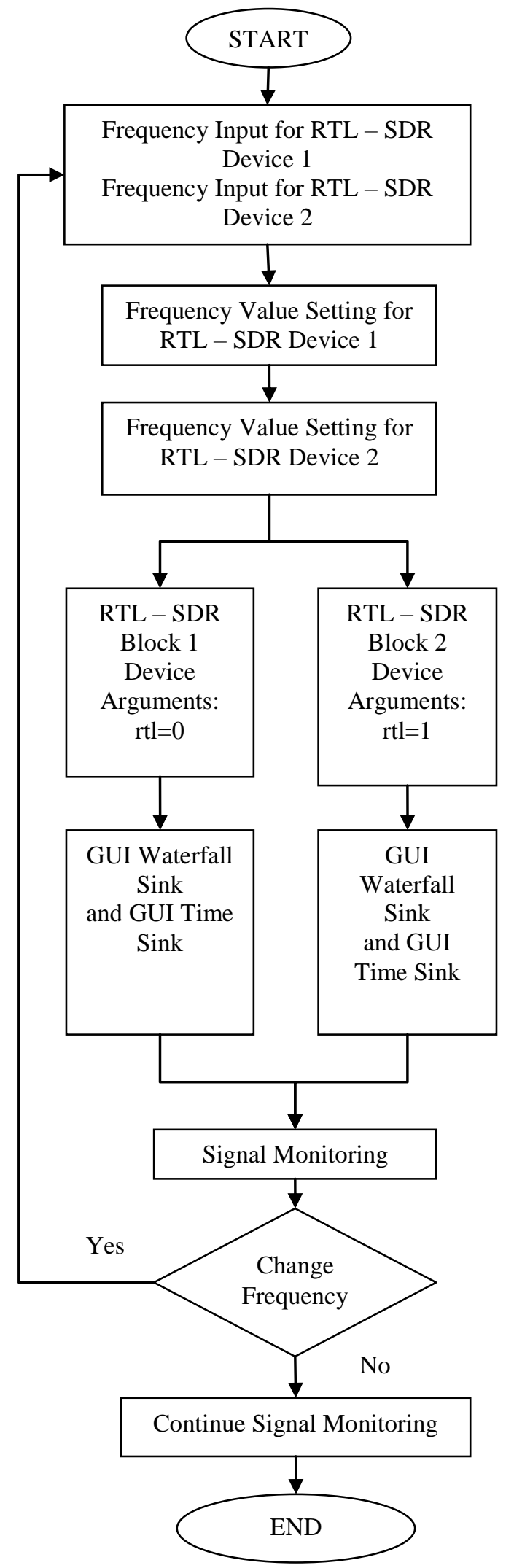

Figure 8 Flowchart for two RTL - SDR detection system 


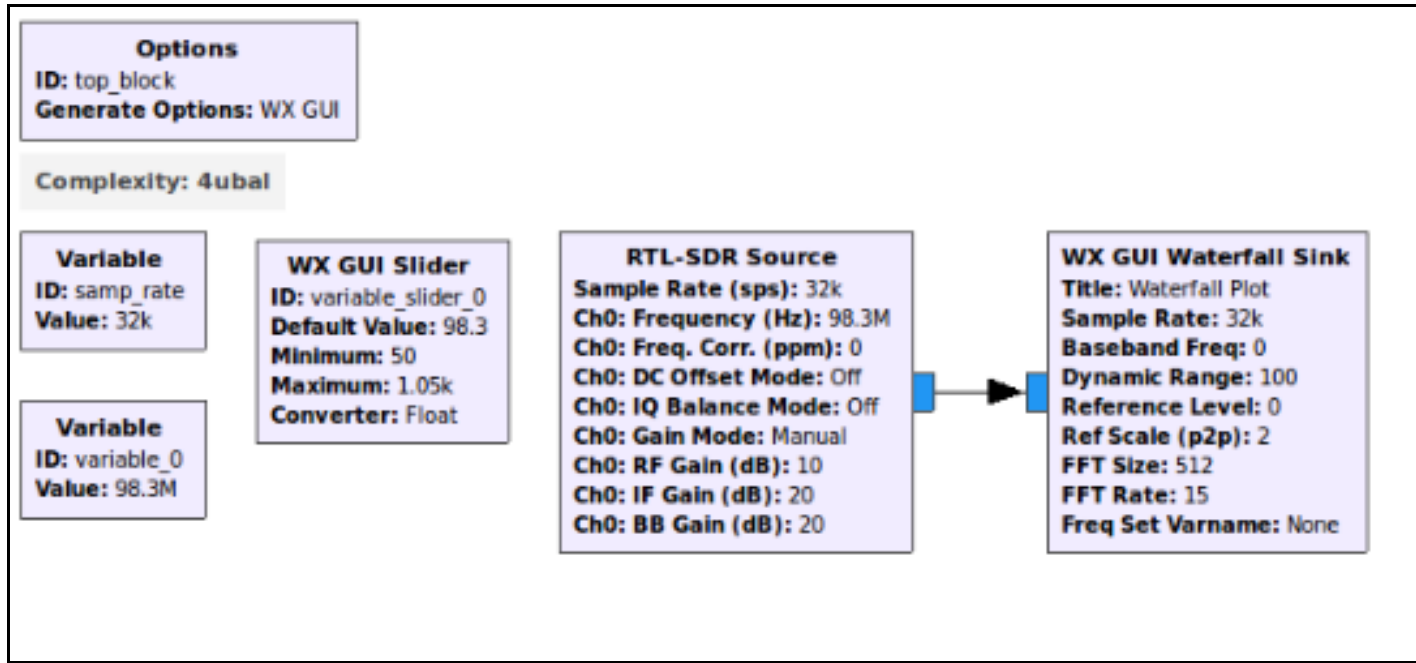

Figure 9 GNU Radio Model for Single RTL - SDR Signal Detection System

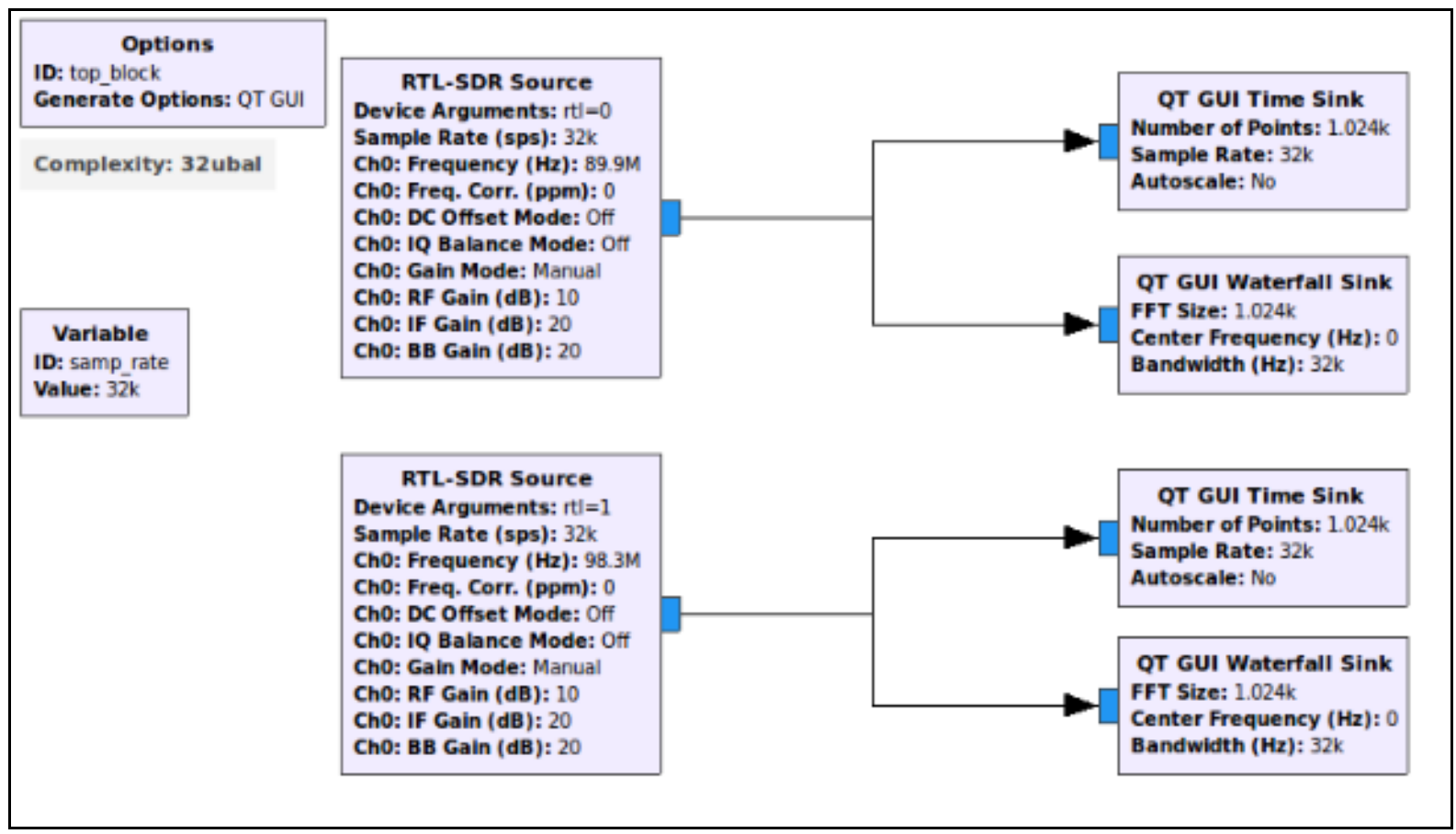

Figure 10 GNU Radio Model for Two RTL - SDR Detection System

\section{TESTING THE SYSTEM USING LOW COST FM TRANSMITTER}

In testing the RTL - SDR and GNU Radio based system we have used low power FM transmitter for transmitting signals. The signal presence was detected by the designed system. In GNU Radio by adding de-modulation blocks the signal can also be de-modulated, which will provide extra feature to recover original message signal. Frequency modulation is that type of angle modulation in which the instantaneous frequency $\omega_{\mathrm{i}}$ is varied linearly with a message or baseband signal $\mathrm{x}(\mathrm{t})$ about an un modulated carrier frequency $\omega_{\mathrm{c}}$, the immediate value of angular frequency $\omega_{i}$ will be equal to the carrier frequency $\omega_{c}$ plus a time changing component proportional to the baseband signal $x(t)$.

Instantaneous frequency is given by

$\omega_{\mathrm{i}}=\omega_{\mathrm{c}}+\mathrm{k}_{\mathrm{f}} \cdot \mathrm{x}(\mathrm{t})$

Where $\mathrm{k}_{\mathrm{f}}$ is proportionality constant and it is known as frequency sensitivity, expressed in Hz/Volt.

The un-modulated carrier signal is

$$
\begin{aligned}
& \mathrm{c}(\mathrm{t})=\mathrm{A} \cos \left(\omega_{\mathrm{c}} \mathrm{t}+\theta_{0}\right) \ldots \\
& \mathrm{c}(\mathrm{t})=\mathrm{A} \cos \phi \ldots \ldots \ldots \ldots \ldots
\end{aligned}
$$

where $\phi=$

$\phi$ is total phase angle of the un-modulated carrier. $\phi_{\mathrm{i}}$ be the instantaneous phase angle of the modulated signal. 
On frequency modulation amplitude A must remain constant and only angle $\phi$ will change.

Hence the expression of frequency modulated wave will be,

$\mathrm{s}(\mathrm{t})=\mathrm{A} \cos \phi \mathrm{i}$

$\phi=\omega_{\mathrm{c}} . \mathrm{t}+\theta_{0}$ from (4).

On differentiation we get

$\frac{d \phi}{d t}=\omega_{c}$

or, we may write the expression for instantaneous phase $\phi_{\mathrm{i}}$ as

$\phi_{\mathrm{i}}=\int \omega_{\mathrm{i}} d t$

Where $\phi_{\mathrm{i}}$ is the instantaneous frequency of frequency modulated sine wave

Putting value of $\omega_{i}$ in (8) from (1) we get

$$
\phi_{\mathrm{i}}=\int\left[\omega_{c}+k_{f} \cdot x(t)\right] d t
$$

Putting the value of $\phi_{\mathrm{i}}$ in (5) we get the expression for frequency modulated wave

$$
s(t)=A \cos \left[\omega_{c} t+k_{f} \int x(t) d t\right]
$$

If the phase angle of the un modulated carrier is taken at $\mathrm{t}=0$, then the limit of integration in equation will be from 0 to $t$.

In this case the expression will be,

$s(t)=A \cos \left[\omega_{c} t+k_{f} \int_{0}^{\pi} x(t) d t\right]$

Which is the required general expression for FM wave. The above expression describes Frequency Modulation basic mathematics. The FM Transmitter used performs modulation of applied signal and it transmits the signal. In testing the signal was not demodulated only FM signal graph and waterfall plots were obtained as given in result section of this paper.

\section{RESULT}

The designed Wireless Transmission Detection and Monitoring System performance is tested for FM Transmission Signals and DVB Signals. The result and detail for FM Signals given below.

Table 1 Details about results obtained for various

\begin{tabular}{|l|l|l|l|}
\hline S.No. & $\begin{array}{l}\text { Signal Type } \\
\& \\
\text { Frequency }\end{array}$ & Result & Remarks \\
\hline 1 & $\begin{array}{l}\text { FM Signal } \\
(88-108 \\
\text { MHz })\end{array}$ & $\begin{array}{l}\text { Able to } \\
\text { detect / } \\
\text { monitor }\end{array}$ & $\begin{array}{l}\text { Both } \\
\text { RTL - } \\
\text { SDR } \\
\text { devices } \\
\text { able to } \\
\text { detect. }\end{array}$ \\
\hline 2 & DVB & Able to & Both \\
\hline
\end{tabular}

\begin{tabular}{|l|l|l|l|}
\hline & Signals (400 & detect / & RTL - \\
$-700 \mathrm{MHz})$ & monitor & $\begin{array}{l}\text { SDR } \\
\text { devices } \\
\text { able to } \\
\text { detect. }\end{array}$ \\
\hline
\end{tabular}

(i) FM Station Signal and Low Power FM Transmitter Signal

FM Broadcast Station Signals successfully detected by the system, at the same time second RTL - SDR Source tuned at Low Power FM Transmitter Frequency and corresponding Waterfall Diagram is shown in the figure 11 .

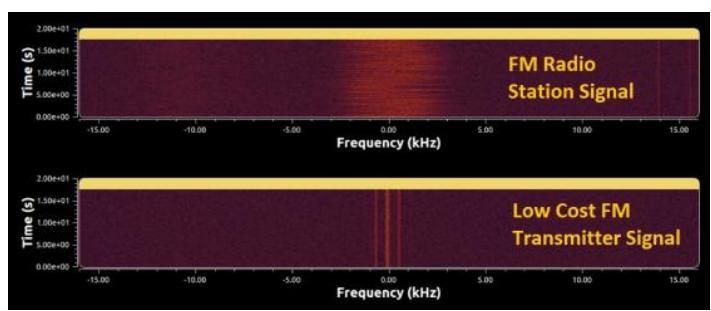

Figure 11 Waterfall graph for FM signal

(ii) FM Signal detection using Single RTL - SDR Design

One RTL - SDR Block based Single Frequency Monitoring System is tuned to local FM Station and the system successfully able to detect. Waterfall Graph for the FM Signal is given in the figure 12.

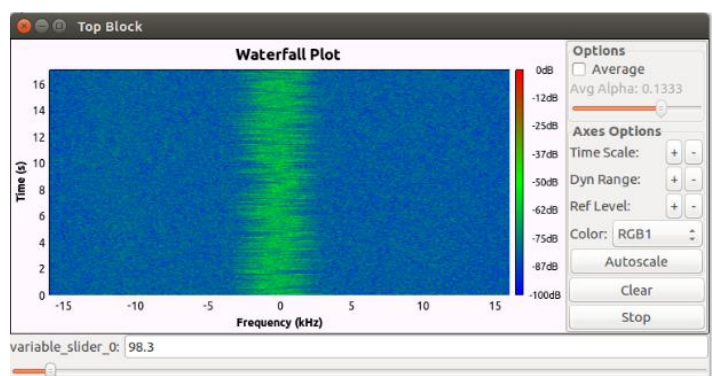

Figure 12 Waterfall plot for single RTL - SDR System

(iii) FFT Plots for two RTL - SDR signals

Two RTL - SDR Source based model is also tested for obtaining FFT plots for user defined frequency. The FFT output for two detected signals is shown in figure 13 .

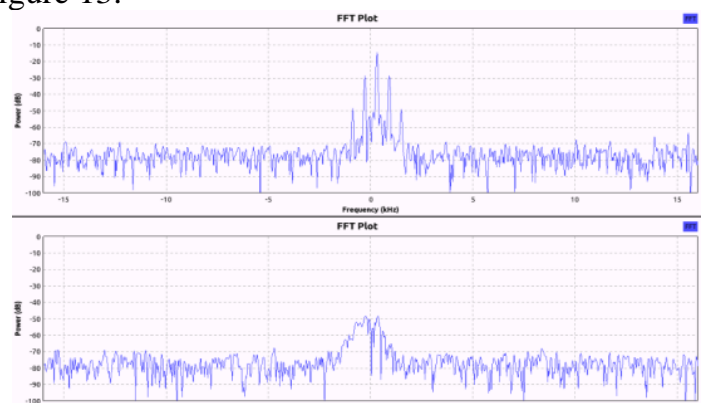

Figure 13 FFT Plot for two RTL - SDR System 


\section{V.FUTURE SCOPE AND APPLICATIONS}

(a.) The proposed SDR based model can be used to monitor low frequency signals in range of Few $\mathrm{KHz}$ to $25 \mathrm{MHz}$ by using suitable up-converter systems. The range below $25 \mathrm{MHz}$ is not supported by RTL SDR hardware.

(b.) The proposed model design is useful in performing transmission monitoring in laboratory environment at very low cost.

(c.) The system is useful for developing hand held devices for monitoring applications.

(d.) The system can be used to monitor wireless control signal for drones in the supporting frequency range.

(e.) The proposed model with two RTL - SDR can be used to detect two different signals at the same time. The system is capable to monitor multiple signals simultaneously.

(f.) Radio signal demodulation capability can also be added to the system.

\section{CONCLUSION}

The RTL - SDR device based system is low cost signal detection and monitoring alternative. The system can be used with mobile devices. The designed system can function with other SDR hardware. The system is helpful in detection and monitoring signals at laboratory environment for various $R \& D$ applications. The overall cost of the system is very less as compared to other SDR systems [6], [7]. Few signal processing blocks used in the model in order to keep the design simple for testing purposes. Various other GNU Radio blocks can be added in order to further process the signal. The model using GNU Radio is able to successfully detect and monitor signal in supported frequency range. The model can also able to demodulate signal by adding suitable signal demodulation blocks. The usage of two RTL - SDR hardware and performing simultaneous signal monitoring gives extra options to work on two different signals at the same time. GNU Radio is also compatible with advanced SDR hardware. The designed system can also work with other SDR hardware by making suitable changes in design.

\section{REFERENCES}

[1] Vijendra Singh Tomar and Vimal Bhatia, “ Low Cost and Power Software Defined Radio using Raspberry $\mathrm{Pi}$ for Disaster Effected Regions" , 2015 - Second International Symposium on Computer and the Internet, Springer, pp $401-404$.

[2] E. G. Sierra and G.A. Ramirez Arroyave, "Low cost SDR spectrum analyzer and analog radio receiver using GNU radio, raspberry Pi2 and SDR-RTL dongle", 2015 - Communications (LATINCOM), 2015 7th IEEE Latin-American Conference, pp $1-6$.
[3] Devarpita Sinha, Anish Kumar Verma and Sanjay Kumar, "Software Defined Radio: Operation, Challenges and Possible Solutions", 2016 - Intelligent Systems and Control (ISCO), 2016 10th International Conference, pp $1-5$.

[4] Robert W. Stewart, Louise Crockett, "A Low Cost Desktop Software Defined Radio Design Environment Using MATLAB, Simulink and the RTL-SDR.", IEEE Communication Magazine, 2015, pp 64 71.

[5] Boonyarit Uengtrakul, "A cost efficient software defined radio receiver for demonstrating concepts in communication and signal processing using Python and RTL-SDR", Digital Information and Communication technology and it's Applications (DICTAP), 2014 Fourth International Conference, pp 394 - 399.

[6] A. Fanan, "Comparison of Spectrum Occupancy Measurements using Software Defined Radio RTL-SDR with a Conventional Spectrum Analyzer approach", $23^{\text {rd }}$ Telecommunications forum TELFOR 2015, IEEE, pp 200 - 203.

[7] Sruthi M B, Abirami M, Akhil Manikkoth and Gandhiraj R ; Soman K P, "Low cost digital transceiver design for Software Defined Radio using RTL-SDR", Automation, Computing, Communication, Control and Compressed Sensing (iMac4s), 2013 International Multi-Conference, pp $852-855$.

[8] Michael Wegerson, Jeremy Straub, Sima Noghanian, Ronald Marsh, "Advancement of Software Defined Radio (SDR) for the Open Orbiter Project", SelectedWorks of Jeremy Straub, http://works.bepress.com/jeremy_straub/

[9] Michael Wegerson, Jeremy Straub, Sima Noghanian, "Work on a Software Defined Radio (SDR) for a CubeSat-Class Spacecraft", SelectedWorks of Jeremy Straub, http://works.bepress.com/jeremy_straub/ 